\title{
Load Distribution Calculation of Flexible Ball Bearing with Elliptical Cam Wave Generator
}

\author{
$\mathrm{Li} \mathrm{Li}^{1, \mathrm{a}}$, Bindi You ${ }^{1, \mathrm{~b}}$ and Jianmin Wen ${ }^{1, \mathrm{c}}$ \\ ${ }^{1}$ School of Naval Architecture and Ocean Engineering, Harbin Institute of Technology, \\ Weihai, 264209, China \\ alilymechanical@163.com, byoubindi@hit.edu.cn, cwenjm@hitwh.edu.cn
}

Keywords: Flexible ball bearing, Mechanical model, Load distribution.

\begin{abstract}
Flexible ball bearing is used as a component in wave generator of a harmonic drive gear system. During the revolution of the flexible bearing, the races of the bearing are subjected to elastic deformation by the shape of the elliptical cam. Thus, a reasonable mechanical model is established based on the analysis of the deformation and stress of the flexible bearing. Further, an analytical model of circular ring under the action of single force is established. Then a load distribution calculation model of the flexible bearing is transformed into linear equations by using the shell theory and the superposition principle.
\end{abstract}

\section{Introduction}

Flexible bearing is a thin section ball bearing, which has an inner ring and an outer ring laid on an inner circumferential surface, and a ball body radially held between the inner and outer rings. They can produce a required function when assembled with an elliptical cam. The controlled deformation of the flexible ball bearing makes the load distribution become more complex. The main challenge of the design to flexible ball bearing is that the rings must be strong enough to carry the load that applying on the ring, so it is necessary to figure out its load distribution. K. X. Le and Y. W. Shen developed an analytical model for calculation of flexible bearing and wave generator [1,2]. Later, Gabriel Cavallaro et al. presented an analytical method to account for the structural deformation of the rings based on Roark's formulas [3]. Ostapski, W. et al. made an analysis of stress state of flexspline and ball bearing in harmonic drive by using FEM method [4]. Z. P. Tang et al. had done the 3D contact analysis for deep groove ball bearing on ANSYS [5]. Recently, Alexandre, D. et al. built a nonlinear plan dynamic model for cylindrical bearings, which can predict the interaction forces between the retainers and the rolling elements [6]. J. W. Zhen et al used PATRAN to make flexibility treatment on the parts of deep groove ball bearing and established the multi-flexibility body rigid-flexible coupling model [7]. Through these studies, they studied stress, strain and friction stress among the inner ring, outer ring and cage.

\section{The Primary Curve of the Flexspline}

The inner ring race is to mounted on the elliptical cam with dimension. The sketch of an elliptical cam is shown in Fig. 1. The major axis semi-diameter of the elliptical cam is $a=r_{b}+w_{0}$, where $r_{b}$ represents the radius of the inner ring, and $w_{0}$ represents the maximum radial displacement of the flexspline. The minor axis semi-diameter can be determined by the condition that the cam's circumference is equal to the inner ring's, suppose the circumference of the elliptical cam is approximately $\pi(a+b)$, then the minor axis semi-diameter can be obtained by $b=2 r_{b}-a=r_{b}-w_{0}$. So the parameter equation of the curve $H$ of the elliptical can be expressed as:

$$
\begin{aligned}
& x_{H}=b \sin \phi_{H} \\
& y_{H}=a \cos \phi_{H}
\end{aligned}
$$


The polar equation of $H$ can be expressed as:

$$
\rho_{H}=\frac{a b}{\sqrt{a^{2} \sin ^{2} \phi_{H}+b^{2} \cos ^{2} \phi_{H}}}
$$

Where $\Phi_{H}$ represents the rotational angle of cam.

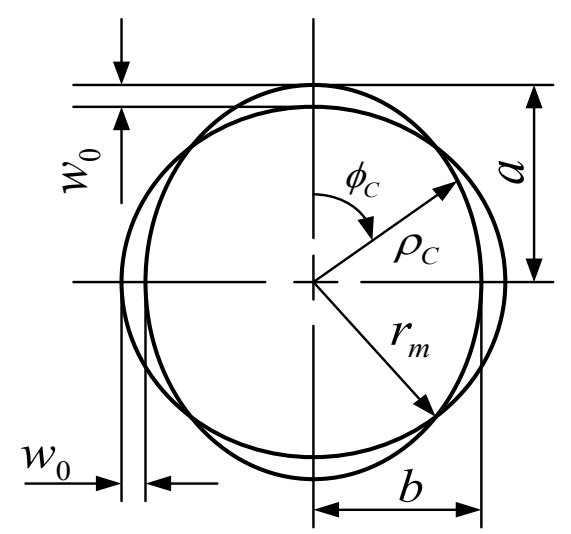

Fig. 1 Schematic sketch of an elliptical cam.

When the generator has been assembled into the shell of the flexspline, the deformed original curve $C$ of the flexspline is considered to be equidistant of the outer curve of $H$.

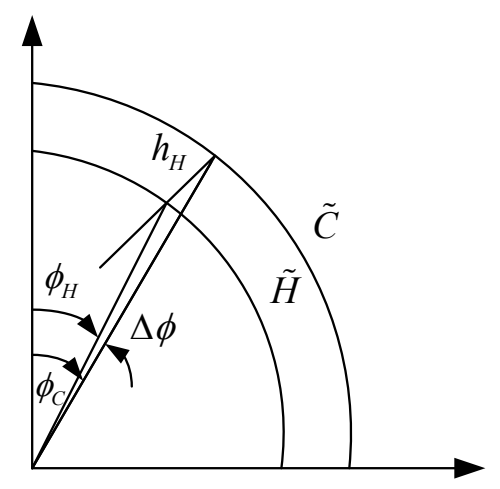

Fig. 2 Sketch of the geometric relationship of $C$ and $H$.

From the geometric relationship in Fig. 2 above, the primary curve $C$ of the flexspline in cartesian coordinate can be expressed as:

$$
x_{C}=b \sin \phi_{H} \pm h_{H}\left|\frac{-a \sin \phi_{H}}{\sqrt{\left(b \cos \phi_{H}\right)^{2}+\left(-a \sin \phi_{H}\right)^{2}}}\right|
$$

where the sign is positive if $0^{\circ}<\varphi_{H}<180^{\circ}$, and the sign is negative if $180^{\circ}<\varphi_{H}<360^{\circ}$, and:

$$
y_{C}=a \cos \phi_{H} \pm h_{H}\left|\frac{b \cos \phi_{H}}{\sqrt{\left(b \cos \phi_{H}\right)^{2}+\left(-a \sin \phi_{H}\right)^{2}}}\right|
$$

where, the sign is positive if $0^{\circ}<\varphi_{H}<90^{\circ}$ or $270^{\circ}<\varphi_{H}<360^{\circ}$, and the sign is negative if $90^{\circ}<\varphi_{H}$ $<270^{\circ} . h_{H}$ is the normal distance between $H$ and $C . R_{b}$ represents the radius of the outer ring of the flexible bearing, $\delta$ represents the thickness of the flexspline gear ring respectively. 
The polar equation of $C$ can be expressed as:

$\rho_{C}=\sqrt{x_{C}^{2}+y_{C}^{2}}$

Then the radial displacement of the primary curve $C$ can be expressed as:

$w_{c}(\phi)=\rho_{C}-R_{b}-0.5 \delta$

\section{Curve Equation under Single Force Action on Ring}

A calculation model under single force action on ring is established as shown in Fig. 3, since the number of the rolling element in the flexible ball bearing used in the harmonic drive is odd, which means there can be one ball at most in the direction of the diameter. Then the solution equations of the static load distribution can be obtained by the superposition of the calculation model mentioned above.

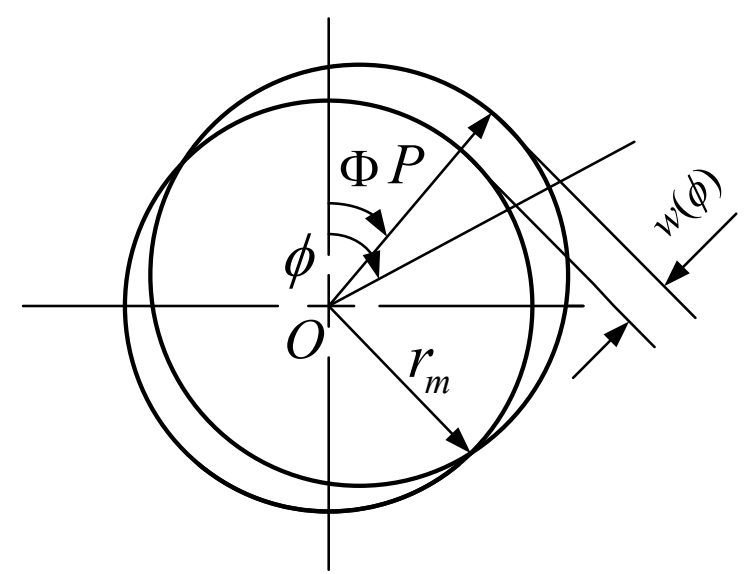

Fig. 3 A calculation model under single force action on ring.

The curved surface of cylindrical flexspline with double wave can be spread into a plane, which simplifies the 3D deformation of the cylinder into the plane problem. The cross section in Fig. 3 is locate at the middle in width of the flexible bearing.

The strain energy of the shell can be obtained from [8], which can be expressed as the following differential equation:

$$
\frac{d^{2} w}{d \varphi^{2}}+w=-\frac{M_{P}}{D\left(1-v^{2}\right)} r_{m}{ }^{2}
$$

where, $w$ represents the radial displacement, $r_{m}$ represents the radius of the neutral surface of the flexspline respectively; $M_{p}$ denotes the bending moment that leads to the circumference's curvature changes in the cross section; $v$ denotes the Poisson's ratio of the flexspline's material; $D$ represents the equivalent stiffness of the flexspline and the outer ring of the ball bearing, it can be calculated by the following formula.

$$
D=\frac{E}{\left(1-v^{2}\right)} J_{P}
$$

where, $E$ denotes the modulus of elasticity; $J_{p}$ denotes the equivalent moment of inertia.

According to the reference [2], the strain energy of the shell is:

$$
V=\int_{0}^{2 \pi} \frac{M_{P}{ }^{2} r_{m}}{2 D\left(1-v^{2}\right)} d \phi
$$


Substitute (8) into the Eq. (10), then the strain energy becomes:

$$
V=\frac{D\left(1-v^{2}\right)}{2 r_{m}^{3}} \int_{0}^{2 \pi}\left(\frac{d^{2} w}{d \varphi^{2}}+w\right)^{2} d \phi
$$

The radial displacement $w$ can also be expressed as:

$$
w=\sum_{n=1}^{\infty}\left(a_{n} \sin n \phi+b_{n} \cos n \phi\right)
$$

where, $a_{n}$ and $b_{n}$ are undetermined coefficient. Substitute (12) into (11), then simplify the equation, the following equation is obtained:

$$
V=\frac{\pi D\left(1-v^{2}\right)}{2 r_{m}{ }^{2}} \sum_{n=2}^{\infty}\left(n^{2}-1\right)^{2}\left(a_{n}^{2}+b_{n}^{2}\right)
$$

The applied force only work in the place of $\phi=\Phi$, according to principle of virtual work:

$$
\begin{aligned}
& \frac{\partial V}{\partial a_{n}} \Delta a_{n}=\frac{\pi D\left(1-v^{2}\right)}{r_{m}{ }^{3}}\left(n^{2}-1\right)^{2} a_{n} \Delta a_{n}=P \Delta a_{n} \sin n \phi \\
& \frac{\partial V}{\partial b_{n}} \Delta b_{n}=\frac{\pi D\left(1-v^{2}\right)}{r_{m}{ }^{3}}\left(n^{2}-1\right)^{2} b_{n} \Delta a_{n}=P \Delta b_{n} \cos n \phi
\end{aligned}
$$

$a_{n}$ and $b_{n}$ can be solved by Eq. (14) and (15), then both them will be substituted into (12) and be simplified:

$$
w=\frac{P r_{m}{ }^{3}}{\pi D\left(1-v^{2}\right)} \sum_{n=2}^{\infty} \frac{\cos n(\phi-\Phi)}{\left(n^{2}-1\right)^{2}}
$$

\section{Mechanical Model of Flexible Ball Bearing}

The mechanical model of the flexible ball bearing is a circular ring that has the same stiffness with the real system. A total of $N$ stress points are the $N$ points of action of rolling elements, as shown in Fig. 5. If $\alpha_{b}$ is the angle between the two adjacent rolling elements, then the polar angle of the number $j$ ball is $\phi_{j}=(j-1) \alpha_{b}$. Obviously, the mechanical model in Fig. 5 is the superposition of $N$ models under single force action on ring every $\alpha_{b}$ angles.

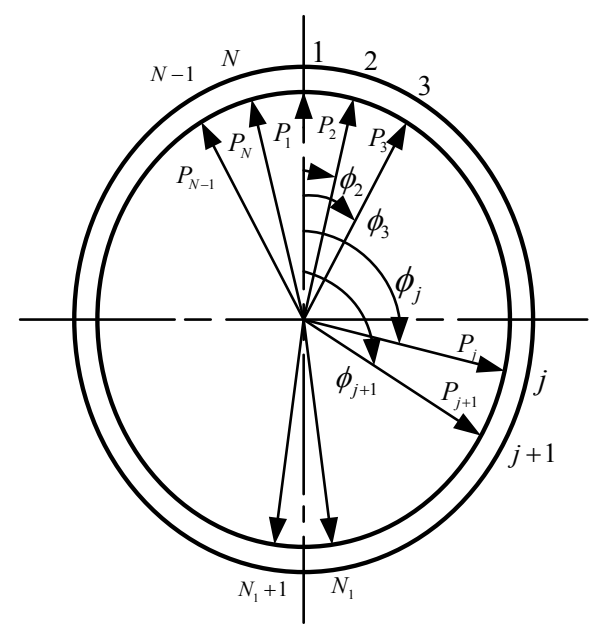

Fig. 4 Model of the flexible ball bearing after deformation. 
Suppose that $w_{i j}$ is the radial displacement of the $i$ point of the ring, and it is generated by the load of the $j$ rolling element, while $w_{c i}$ is the radial displacement of the $i$ point of the curve defined in (7), then the following equation will be obtained:

$$
\sum_{j=1}^{N} w_{i j}=w_{c i},(i=1,2, \cdots, N)
$$

Substitute (16) into the Eq. (17), the mathematical model of the load distribution of the flexible ball bearing will be obtained.

$$
\sum_{j=1}^{N} A_{i j} P_{j}=w_{c i},(i=1,2, \cdots, N)
$$

where:

$$
A_{i j}=\frac{r_{m}{ }^{3}}{\pi D\left(1-v^{2}\right)} \sum_{n=2}^{\infty} \frac{\cos n\left(\varphi_{i}-\varphi_{j}\right)}{\left(n^{2}-1\right)^{2}},(i=1,2, \cdots, N ; j=1,2, \cdots, N)
$$

\section{Model Parameters and Analysis}

The obtained mathematical model of the load distribution of the flexible ball bearing in Eq. (18) is a set of $\mathrm{N}$-dimensional linear equations. The rolling element of the ball bearing can only bear the pressure and cannot bear the tension, while there may be a negative phenomenon in calculating $P_{j}$ of Eq. (18). To handle this negative phenomenon, when the results are obtained for the first time, they should be checked by point, set the point to zero that the absolute value of the negative is the maximal, and note the number of this point. Recalculate the equation after its dimension is reduced by 1-dimension, check by point again, and the cycle repeats itself until the values of $P_{j}$ are no more negative.

The parameters of the flexible ball bearing and the flexspline, and the material properties in the load calculation are shown in Table 1. To compare the calculated results with the results of the wave generator which is equipped with four-force action type cam, it is noted that the main parameters in the Table 1 is almost the same with [1], in this way, it can be proved that the calculation model in reasonable.

Table 1. Parameters in the calculation.

\begin{tabular}{cc}
\hline Ball diameter & $14.288(\mathrm{~mm})$ \\
\hline Number of balls & 23 \\
Bearing width & $25(\mathrm{~mm})$ \\
Poisson's ratio & 0.3 \\
Modulus of elasticity & $210(\mathrm{GPa})$ \\
Bearing outer ring radius & $80(\mathrm{~mm})$ \\
Bearing inner ring radius & $60(\mathrm{~mm})$ \\
Flexspline Neutral Radius & $81(\mathrm{~mm})$ \\
Maximum radial displacement & $0.7(\mathrm{~mm})$ \\
\hline
\end{tabular}

\section{Simulation Results and Discussions}

The simulated results by MATLAB software are shown in Table 2. The load are mainly distributed in the area of 313 47 degrees and 141 219 degrees, the load center of the former area is located at $344 \sim 16$ degrees with the maximal value in the location of 0 degree, and the load center of the latter is located at 172 188 degrees with an equal value. The position is exact where the maximum radial displacement of the ellipse major axis locates at. 
Table 2. Load distribution results of flexible ball bearing.

\begin{tabular}{cccccccccc}
\hline No. & w0 $=0.5$ & $\mathrm{w} 0=0.6$ & $\mathrm{w} 0=0.7$ & $\mathrm{w} 0=0.8$ & No. & $\mathrm{w} 0=0.5$ & $\mathrm{w} 0=0.6$ & $\mathrm{w} 0=0.7$ & $\mathrm{w} 0=0.8$ \\
\hline 1 & 708.39 & 845.30 & 980.75 & 1114.8 & 13 & 635.41 & 766.13 & 898.07 & 1031.2 \\
2 & 655.91 & 782.29 & 907.13 & 1030.5 & 14 & 537.31 & 647.32 & 758.12 & 869.7 \\
3 & 506.80 & 602.78 & 697.00 & 789.4 & 15 & 366.74 & 442.78 & 519.67 & 597.4 \\
4 & 329.14 & 392.76 & 455.72 & 518.1 & 16 & 199.80 & 240.74 & 282.06 & 323.8 \\
5 & 111.63 & 128.29 & 143.16 & 156.3 & 17 & 0 & 0 & 0 & 0 \\
6 & 0 & 0 & 0 & 0 & 18 & 0 & 0 & 0 & 0 \\
7 & 0 & 0 & 0 & 0 & 19 & 0 & 0 & 0 & 0 \\
8 & 0 & 0 & 0 & 0 & 20 & 111.63 & 128.29 & 143.16 & 156.3 \\
9 & 199.80 & 240.74 & 282.06 & 323.8 & 21 & 329.14 & 392.76 & 455.72 & 518.1 \\
10 & 366.74 & 442.78 & 519.67 & 597.4 & 22 & 506.80 & 602.78 & 697.00 & 789.4 \\
11 & 537.31 & 647.32 & 758.12 & 869.7 & 23 & 655.91 & 782.29 & 907.13 & 1030.5 \\
12 & 635.41 & 766.13 & 898.07 & 1031.2 & & & & & \\
\hline
\end{tabular}

In the Table 2 above, the load distribution of two areas mentioned above is not symmetrical because the number of the rolling element is odd, while the load in the place of 172 degree is equal to the load in the place of 188 degree, this is because the balls in this two locations are symmetrical to the major axis. The load in the place of 0 degree is slightly larger than the load in the place of 172 or 188 degree, which is correspond to the rules of radial displacement distribution of the elliptical cam profile. In addition, the load in the area of 78 110 degrees and 250 282 degrees is 0 where the minor axis of the elliptical cam locates, this is because that the radial displacement of the primary curve of the flexspline is negative, which means there is no load applied in this two areas.

Moreover, it can be seen from Fig. 5 that the load of the two stressed area increases as the maximum radial displacement $w_{0}$ increases, and the closer to the center of the load area, the more the corresponding load is added. At the load center, each $0.1 \mathrm{~mm}$ increase in the maximum radial displacement is related to about $133 \mathrm{~N}$ load increase accordingly. While away from the load center, the load of the flexible ball bearing is increased slowly with about 13 to $42 \mathrm{~N}$ increase per $0.1 \mathrm{~mm}$.

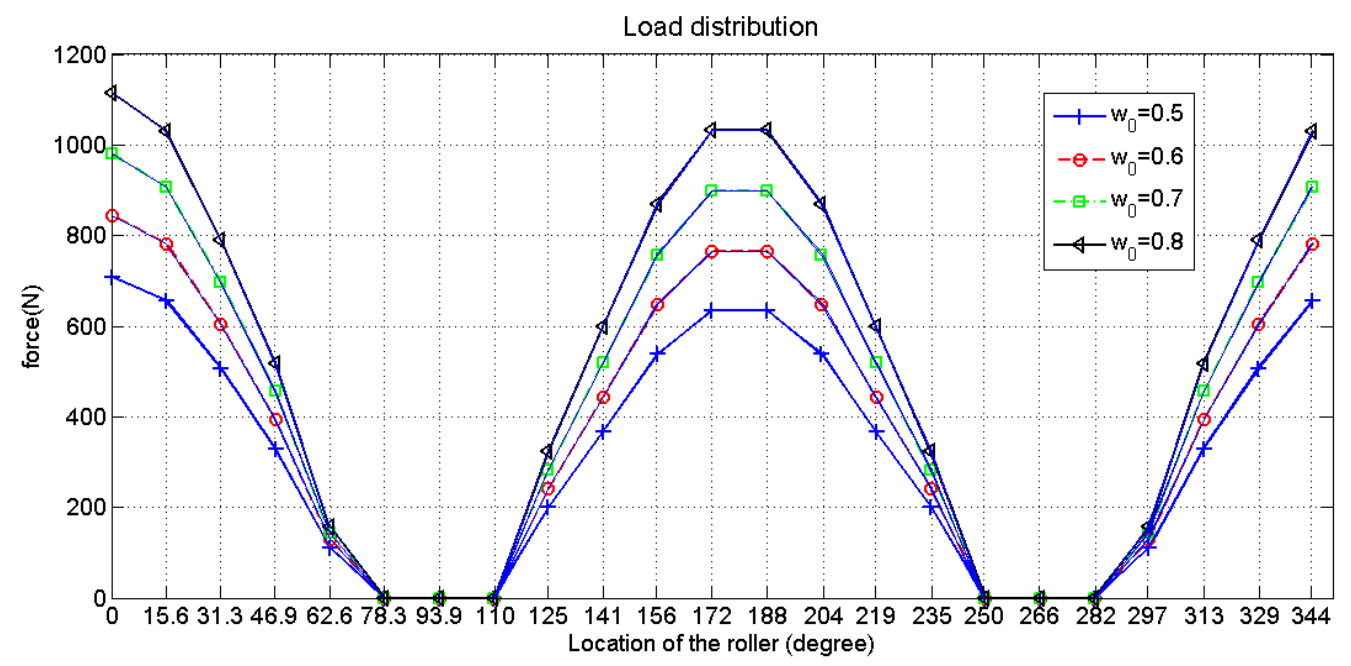

Fig. 5 Calculated load distribution.

\section{Conclusion}

(1) A reasonable mechanical model is established and a group of linear equations is obtained.

(2) The results is consistent with the radial displacement distribution of the elliptical cam.

(3) The load of the two forced area increases as the maximum radial displacement increases. 


\section{Acknowledgment}

This paper is based on Projects (No. 51575126) supported by National Natural Science Foundation of China, and supported by Discipline Construction Guide Foundation in Harbin Institute of Technology at Weihai (Grant Nos. WH20150108 and WH20140105), and the Projects (HIT. NSRIF. 2015110) supported by Natural Scientific Research Innovation Foundation in Harbin Institute of Technology.

\section{References}

[1] K. X. Le and Y. W. Shen, Load distribution on the wave generator of harmonic gear transmission, Mech. Sci. Technol. (1990) 38-45.

[2] K.X. Le, Y. X. Quan, G. R. Zhou, Z. N. Chen and M. J. Zhang, A new method for calculating load distribution on harmonic rolling bearing, J. Zhejiang U. (Eng. Edition), (1994) 396-404.

[3] G. Cavallaro, D. Nelias and F. Bon, Analysis of High-Speed Intershaft Cylindrical Roller Bearing with Flexible Rings, Tribol. T. 48(2) (2005) 154-164.

[4] W. Ostapski and I. Mukha, Stress state analysis of harmonic drive elements by FEM, B. Pol. Acad. Sci. Tech. Sci. 55(1) (2007) 115-123.

[5] Z. P. Tang and J. P. Sun, The contact analysis for deep groove ball bearing based on ANSYS, 2011 International Conference on Power Electronics and Engineering Application Procedia Engineering . (2011), p. 423-428.

[6] L. Alexandre, D. Nelias and C. Defaye, Nonlinear dynamic analysis of cylindrical roller bearing with flexible rings, J. Sound Vib. 325(1) (2009) 145-160.

[7] J. W. Zhen, L. L. Cui and X. Chen, Mechanics Modeling for Bearing Rigid-Flexible Coupling Multi-Body System Based on ADAMS, Appl. Mech. Mater. 364 (2013) 124-128.

[8] S. P. Timoshenko and J. M. Gere, Elastic stability theory. F. F. Zhang translation. Beijing: Science Press, 1965 